\section{Spectra and Efficiency of Binary Codes Without DC}

\section{J. N. FRANKLIN AND J. R. PIERCE}

\begin{abstract}
In digital transmission of binary $(+1,-1)$ signals it is desirable that the stream of pulses which constitutes the signal have no dc, that is, that the power spectrum go to zero at zero frequency. It is desirable that, for a given efficiency or entropy, the spectrum rise slowly with increasing frequency. We have obtained the spectrum for selected blocks with equal numbers of plus ones and minus ones. For a given efficiency, this is better than the spectrum obtained by Rice, using the Monte Carlo method, for block encoding using polarity pulses. An algorithm given by Schalwijk should allow simple encoding into selected blocks.
\end{abstract}

The spectra (hitherto unpublished) and the efficiencies of two schemes of encoding which give pulse streams with no dc are compared, and it is shown that one is superior to the other.

In digital transmission the power spectrum of the pulse train can be shaped through the introduction of redundancy. Usually in so doing the spectrum is made zero at some particular frequency and small near that frequency. Frequencies commonly chosen are 0 and $N / 2$ (the Nyquist bandwidth). Practical reasons for doing this include [1]

1) ( $f=0)$ avoidance of interference with audio and other low-frequency signals;

2) $(f=0)$ signals will pass through transformers;

3) $(f=0$ or $N / 2)$ allows transmission of a carrier or timing wave;

4) ( $f=0$ and $f=N / 2)$ reduces intersymbol interference due to bad phase and gain at band edges.

Another reason sometimes given is that redundancy can allow error detection or correction. From a practical point of view this seems a dubious advantage in transmission systems in which repeaters must be cheap and simple. It is more plausible that error control should be supplied by the user according to his needs. Detection of gross format errors as an indication of faulty operation is of course reasonable.

Happily, it is possible to use a technique of interleaving to make the spectrum zero at $N / 2$ (or at other fractions of $N$ ) if it is zero at zero [1]. Thus, schemes which make the spectrum 0 at $f=0$ and low for low frequencies are particularly useful. Many simple and complicated schemes have been proposed $[2]-[5]$.

A desirable encoding scheme is one which 1) is simple to implement and 2) is efficient in reducing the power spectrum over a wide enough frequency range at a small cost in information rate. Thus, in order to evaluate a scheme we need to consider its complexity, its information rate, and its spectrum. Unhappily, it is difficult to calculate spectra and only a few simple spectra are given in the literature [3] .

In the case of binary, $(+1,-1)$ transmission, one very simple scheme might be called the polarity pulse method. In this method, $n-1$ message digits are sent in a block of $n$ digits, either sent as they are or inverted (multiplied by -1). Whether or not the digits have been inverted is communicated by means of a polarity pulse in the block, which may, for instance, be +1 for no inversion and -1 for an inversion. The polarity pulse is chosen so as to make the absolute value of the sum of all past digits plus those of the block which will be sent as small as possible [1], [6]. (The same technique can be extended to

Paper approved by the Data Communications Committee of the IEEE Communications Society for publication without oral presentation. Manuscript received May 31, 1972 ; revised June. 27, 1972.

The authors are with the Department of Electrical Engineering, CaliThe authors are with the Department of Electrical Eng
fornia Institute of Technology, Pasadena, Calif. 91109. punch a hole anywhere in the spectrum by basing the choice of polarity pulses on a Fourier analysis.)

In unpublished work, Rice has obtained the power spectrum analytically for a random signal stream for a block length of 2 , and he made Monte Carlo calculations for block lengths of $2,4,6,8$ and 16 . The Monte Carlo points lay about the correct curve for $n=2$, but all the Monte Carlo spectra were ragged. We have made estimates by inspection of the fraction of the Nyquist band at which the power spectrum rose to $1 / 2$ of the flat spectrum amplitude for a sequence of random digits; these will be tabulated later.

Another scheme is block encoding using only selected blocks which contain equal numbers of positive and negative pulses. In this case the efficiency or transmission rate $E$, in bits per digit, is given by

$$
E=\frac{1}{n} \log _{2} \frac{n !}{\left(\left(\frac{n}{2}\right) !\right)^{2}}
$$

Here $n$ is the block length. For large values of $n$,

$$
E \doteq 1-\frac{1}{2 n} \log _{2} n+\frac{1}{2 n} \log _{2}\left(\frac{2}{\pi}\right)+0\left(\frac{1}{n^{2}}\right) \text {. }
$$

Fortunately, the power spectrum $p(w)$ can be found as the ensemble average for blocks chosen randomly with equal probabilities. It is derived in the Appendix and is such that

$$
2 \pi P(\omega)=\frac{n}{n-1}\left[1-\frac{1}{n^{2}}\left(\frac{\sin (n \omega / 2)}{\sin (\omega / 2)}\right)^{2}\right]
$$

Here the Nyquist bandwidth corresponds to $\omega=\pi$. Fig. 1 shows the power spectrum for $n=6$.

In comparing this selected block encoding with the encoding considered earlier, we can ask, as a function of $n$, what is the efficiency and at what fraction of the Nyquist bandwidth $(\omega / \pi)$ is $(3)$ equal to $1 / 2$.

In Table I, $n$ is block length, $E$ is efficiency in bits per digit, and $B$ is the fraction of the Nyquist bandwidth at which the power spectrum has risen to 0.5 .

Block lengths have been selected which give approximately the same efficiency. We see that the selected block method requires larger block lengths for a given efficiency but gives a broader band in which the frequency spectrum is low. However, as the block length and efficiency go up, the relative performance of polarity pulse encoding improves.

It is easy to see why the spectrum of the polarity pulse signal is inferior at small block lengths by considering the case of a block length $n=2$. For selected blocks,

$$
\begin{aligned}
1 & \rightarrow+1,-1 \\
-1 & \rightarrow-1,+1 .
\end{aligned}
$$

Each code has the same favorable power spectrum, so the power spectrum is

$$
2 \pi P(\omega)=2 \sin ^{2}(\omega / 2) .
$$

In polarity pulse encoding,

$$
\begin{array}{r}
1 \rightarrow+1,+1 \text { or }-1,-1 \\
-1 \rightarrow-1,+1 \text { or }+1,-1 .
\end{array}
$$

The patterns $+1,-1$ and $-1,+1$ give the same favorable component of spectrum as before, but the patterns $+1,+1$ and -1 , -1 , which must occur in equal numbers, give an inferior component to the spectrum.

Polarity pulse encoding and decoding is very simple and a 


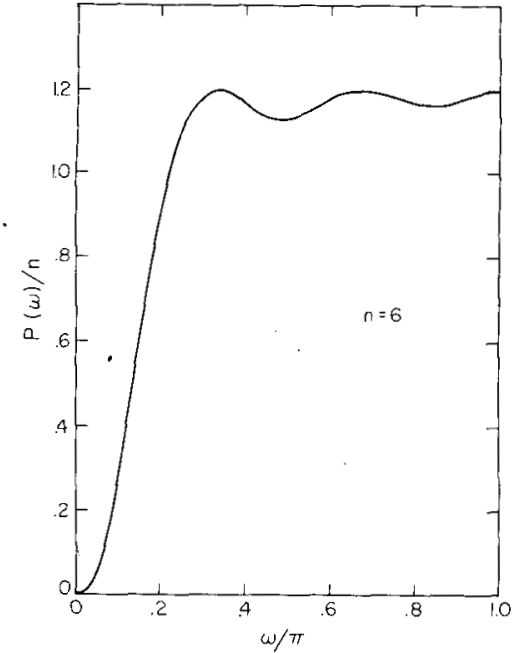

Fig. 1 .

high efficiency can be attained with a moderate block length, but the spectrum is inferior to selected block encoding.

Selected block encoding gives the maximum information rate (assuming that all blocks are chosen randomiy with the same probability) for the block length and the spectrum specified by (3). It is not maxentropic in Slepian's sense [7], because a greater efficiency might be attained for the same spectrum and a longer block. It should be noted that Slepian's mathematical arguments do not apply because the probabilities of blocks with unequal numbers of plus ones and minus ones are zero.

Fortunately, a simple algorithm given by Schalwijk [8] allows the assignment of unique numbers to blocks of equal weight. By using this algorithm it should be possible to go from binary to selected blocks and back to binary with digital circuits of moderate complexity.

\section{APPENDIX}

\section{SPECTRUM OF THE SIGNALS}

Each signal has the form

$$
x(t)=\sum_{k=1}^{n} x_{k} \frac{\sin \pi(t-k)}{\pi(t-k)}
$$

where every $x_{k}= \pm 1$ and where $\Sigma x_{k}=0$. Since

$$
\int_{-\infty}^{\infty} \frac{\sin \pi(t-k)}{\pi(t-k)} \frac{\sin \pi(t-l)}{\pi(t-l)} d t= \begin{cases}1, & k=l \\ 0, & k \neq l\end{cases}
$$

we have the total power

$$
\int_{-\infty}^{\infty} x^{2}(t) d t=n
$$

Using the Fourier transform, we find

$$
x(t)=\int_{-\pi}^{\pi}\left(\frac{1}{2 \pi} \sum_{k=1}^{n} x_{k} e^{i k \omega}\right) e^{-i t \omega} d \omega
$$

and Plancherel's theorem states

$$
\int_{-\infty}^{\infty} x^{2}(t) d t=\int_{-\pi}^{\pi} \frac{1}{2 \pi}\left|\sum_{k=1}^{n} x_{k} e^{i k \omega}\right|^{2} d \omega .
$$

TABLE 1

\begin{tabular}{rccccc}
\hline \hline & \multicolumn{2}{c}{ Selected Blocks } & \multicolumn{3}{c}{ Polarity Pulse } \\
$n$ & $E$ & $B$ & $B$ & $E$ & $n$ \\
\hline 2 & 0.500 & 0.333 & 0.109 & 0.500 & 2 \\
8 & 0.766 & 0.1022 & 0.058 & 0.750 & 4 \\
14 & 0.839 & 0.0603 & 0.041 & 0.833 & 6 \\
22 & 0.883 & 0.0391 & 0.031 & 0.875 & 8 \\
50 & 0.937 & 0.0175 & 0.014 & 0.937 & 16 \\
\hline
\end{tabular}

The power spectrum is defined as the expected value of the last integrand:

$$
P(\omega)=E \frac{1}{2 \pi}\left|\sum_{k=1}^{n} x_{k} e^{i k \omega}\right|^{2}
$$

where $-\pi \leqslant \omega \leqslant \pi$. If $|\omega|>\pi$, we have $P(\omega) \equiv 0$.

In the expectation (7) we must evaluate the autocorrelations $E\left(x_{k} x_{l}\right)$. If $k=l$, we have $x_{k}= \pm 1$, and hence $E\left(x_{k}^{2}\right)=1$. If $k=l$, we have $x_{k}= \pm 1$, and hence $E\left(x_{k}^{2}\right)=1$. If $k \neq l$, we have

$$
\begin{aligned}
E\left(x_{k} x_{l}\right) & =\operatorname{Pr}\left(x_{k} x_{l}=1\right)-\operatorname{Pr}\left(x_{k} x_{l}=-1\right) \\
& =2 \operatorname{Pr}\left(x_{k} x_{l}=1\right)-1 \\
& =2 \operatorname{Pr}\left(x_{k}=x_{l}\right)-1 .
\end{aligned}
$$

Half of the numbers $x_{1}, \cdots, x_{n}$ are +1 , and half are -1 . If $x_{k}=+1,(n / 2)-1$ of the remaining $n-1$ numbers are +1 . Hence, the probability that $x_{l}$ equals +1 , given that $x_{k}=+1$, is the quotient

$$
\frac{\frac{n}{2}-1}{n-1}
$$

Similarly, this quotient equals the probability that $x_{l}=-1$, given that $x_{k}=-1$. In summary, if $k \neq l$,

$$
\operatorname{Pr}\left(x_{k}=x_{l}\right)=\frac{\frac{n}{2}-1}{n-1} \text {. }
$$

Then, (8) implies, if $k \neq l$,

$$
E\left(x_{k} x_{l}\right)=\frac{-1}{n-1} .
$$

The definition ( 7$)$ of $P(\omega)$ implies

$$
2 \pi P(\omega)=\sum_{k=1}^{n} \sum_{l=1}^{n} E\left(x_{k} x_{l}\right) e^{i(k-l) \omega}
$$

Separating the terms with $k=l$, we find

$$
2 \pi P(\omega)=n+\sum_{k \neq l} E\left(x_{k} x_{l}\right) e^{i(k-l) \omega}
$$

Now (10) implies

$$
\begin{aligned}
2 \pi P(\omega) & =n+\sum_{k \neq l} \frac{-1}{n-1} e^{i(k-l) \omega} \\
& =n+\frac{n}{n-1}-\frac{1}{n-1} \sum_{k=1}^{n} \sum_{l=1}^{n} e^{i k \omega} e^{-i l \omega} \\
& =\frac{n^{2}}{n-1}-\frac{1}{n-1}\left|\sum_{k=1}^{n} e^{i k \omega}\right|^{2}
\end{aligned}
$$


But

$$
\left|\sum_{k=1}^{n} e^{i k \omega}\right|=\left|\frac{e^{i n \omega}-1}{e^{i \omega}-1}\right|=\left|\frac{e^{i(n / 2) \omega}-e^{-i(n / 2) \omega}}{e^{i(\omega / 2)}-e^{-i(\omega / 2)}}\right|
$$$$
=\left|\frac{\sin \frac{n}{2} \omega}{\sin \frac{1}{2} \omega}\right| .
$$

Therefore,

$$
2 \pi P(\omega)=\frac{n}{n-1}\left[1-\frac{1}{n^{2}}\left(\frac{\sin \frac{n}{2} \omega}{\sin \frac{1}{2} \omega}\right)\right] .
$$

\section{REFERENCES}

[1] ]. R. Pierce, "Some practical aspects of digital transmission, IEEE

[2] Kpectrum, vol. 5, pp. 63-70, 1968 . Cattermole, Principles of Pulse Code Modulation. London: Illife Books, 1969 .

[3] E. Gorog, "Redundant alphabets with desirable frequency spectrum properties," IBM J. Res. Develop., vol. 12, pp. 234-241, May 1968.

[4] U. Appel and K. Tröndle, "Zusammenstellung und Gruppierung verschiedener Codes für die Übertragung digitalen Signale," Nachrichtentech. Z., vol. 23 pp. 11-16, Jan. 1970 .

[5] H. Kobay ashi, "A survey of coding schemes for transmission or recording of data," IEEE Trans. Commun. Technol., vol. COM-19, pp. 1087-1100, Dec. 1971 .

[6] F. K. Bowers, "Pulse code transmission system," U.S. Patent 2957947 , Oct. 25,1960 .

[7] D. Slepian, "On maxentropic discrete stationary processes," Bell Syst. Tech. J., vol. 51, pp. 629-653, Mar. 1972 .

[8] J. P. M. Schalwijk, "An algorithm for source coding," IEEE Trans. Inform. Theory, vol. IT-18, pp. 395-399, May 1972 .

\section{.An Adaptive Electronic Hybrid Transformer}

\section{STANLEY A. WHITE}

\begin{abstract}
A hybrid transformer is used to couple simultaneously both a transmitter and a receiver to the same end of a telephone line with minimum crosstalk. Under ideal circumstances the transformer provides a cancellation signal at the input terminals of the receiver to exactly cancel the signal induced by the local transmitter. This paper describes a simple electronic device that closely behaves as a hybrid transformer operating under ideal conditions. Rejection on dial-up lines is between $50-80 \mathrm{~dB}$. Adaption time is on the order of $50 \mathrm{~ms}$.
\end{abstract}

\section{INTRODUCTION}

The purpose of a hybrid transformer in a data modem is to provide some degree of isolation between the signals that are simultaneously transmitted and received over a two-wire line. The transformers are designed to work with a nominal line impedance, which limits the effectiveness of the transformer. This paper presents a self-balancing electronic configuration to equalize the effects of the varying load impedance that the transmission line presents to the modem.

A partial block diagram of the device is shown in Fig. 1. In Fig. 2 are illustrated the waveform from the transmitter $e_{t}$; that same signal phase shifted $e_{t p}$; the signal as seen on the

Paper approved by the Data Communications Committee of the IEEE Communications Society for publication without oral presentation. Manuscript received April 24, 1972 ; revised June 12, 1972.

The author is with the Autonetics Division of North American Rockwell, Anaheim, Calif. transmission line $e_{o}$ consisting of both the high-amplitude outgoing signal and the low amplitude incoming signal; and the signal at the receiver terminals $e_{r}$ consisting ideally only of the incoming signal.

\section{SIMPLIFIED APPROACH}

The transmission line is, of course, a distributed-parameter load. Several lumped-parameter approximations were mechanized in early trials. Surprisingly, however, a suitable line-load model is a simple first-order parallel $R C$ network. This permits the system block diagram to be reduced to the simple configuration illustrated in Fig. 3.

The parameter adjustment servo may be mechanized by demodulating $e_{r}$ with respect to $e_{t}$ and $e_{t p}$ in order to determine the instantaneous errors, then integrating these signals to set the phase-shift gain $K_{1}$ and the signal-amplitude gain $K_{3}$ as shown in Fig. 4.

The signal provided by the transmitter $e_{t}$ is operated on by two gains $K_{1}$ and $K_{2}$ (the first of which also contains a differentiator), which in turn feed a line driver of gain $K_{3}$ whose unloaded output is

$$
E_{D}=E_{t} K_{3}\left(K_{1} s+K_{2}\right),
$$

where capital letters are used to designate the Laplace transform of those time variables designated by lower-case letters. The signal at the telephone line terminals is

$$
e_{o}=e_{o t}+e_{r}
$$

The signal that is supplied by the transmitter to the telephoneline load (whose assumed admittance is $Y_{L}={ }_{s} C_{L}+1 / R_{L}$ ) is

$$
\begin{aligned}
E_{0 t} & =E_{D} \frac{R_{L}}{R_{0} R_{L} C_{L} s+R_{0}+R_{L}} \\
& =E_{t} R_{L} K_{3} \frac{K_{1} s+K_{2}}{R_{0} R_{L} C_{L} s+R_{0}+R_{L}} \\
& =E_{t} \frac{R_{L} K_{2} K_{3}}{R_{0}+R_{L}} \frac{\frac{K_{1}}{K_{2}} s+1}{\frac{R_{0} R_{L} C_{L}}{R_{0}+R_{2}} s+1}
\end{aligned}
$$

The signal at the output of the receiver amplifier is

$$
e_{r}=e_{0 t}-e_{t}+e_{L}
$$

where $e_{L}$ is the incoming signal on the phone line. We would like to make $e_{0 t}=e_{t}$ so that $e_{r}=e_{L}$. In order to mechanize this, the signal $e_{r}$ is monitored by a parameter adjustment servo to accomplish two tasks.

1) The first task is to phase shift the signal $e_{t}$ such that the transmitted signal $e_{0 t}$ is in phase with $e_{t}$. In other words, the phase lead of the signal $e_{D}$ offsets the lagging effect caused by the line-load impedance.

2) The second task is to adjust the gain of the transmitted signal such that the amplitude of the transmitted signal $e_{0 t}$ is the same as the amplitude of the transmitter input to the hybrid $e_{t}$.

In other words, the output signal is sampled and phase detected using the output of the quadrature channel amplifier as a reference. The resulting error signal is fed back to adjust the gain of $K_{1}$ to force the numerator and denominator time constants of (2) to be equal. The output voltage is then

$$
e_{0 t}=e_{t} K_{2} K_{3} \frac{R_{L}}{R_{0}+R_{L}}
$$

